\title{
THE URINARY PHENOLSULPHONPHTHALEIN (PHENOL RED) EXCRETION TEST IN HYDROCEPHALUS
}

\author{
BY \\ K. M. LAURENCE \\ From The Hospital for Sick Children, Great Ormond Street, London
}

(RECEIVED FOR PUBLICATION APRIL 10, 1957)

The classification of hydrocephalus into communicating and non-communicating types is still largely based on the dye test introduced by Dandy and Blackfan in 1914 and 1917 . They used the appearance of dye in the spinal fluid after its injection into one of the lateral ventricles as the criterion.

In the same papers Dandy and Blackfan described a quantitative urinary dye excretion test. They showed that in cases of hydrocephalus the amount of phenol red which is excreted in the urine in a two-hourly period after its intraventricular or intrathecal injection is less than in cases with a normal cerebrospinal fluid pathway. The excretion patterns fell into two fairly distinct groups according to whether a communication did or did not exist, though it was suggested that there might be an intermediate group with a 'partial' communication. The reduced excretion results were assumed to reflect the reduction of the available absorptive surface in the subarachnoid space. Their observations were based on a small series of cases and in only 15 of their 26 patients was the anatomical site of the blockage causing the hydrocephalus known.

This work was shortly overshadowed by the introduction of pneumoencephalography with its wider application to other conditions (Heuer and Dandy, 1916; Dandy, 1919). The present study was undertaken to explore and develop the possibilities of the urinary dye excretion test in hydrocephalus, and this report is based on 165 dye tests carried out on 101 patients as shown in Table 1. In all these cases the anatomical site of the block has been ascertained, either from satisfactory pneumoencephalography or at post-mortem examination. Tests, which were unsatisfactory because of incomplete urine collection, poor urinary output or some other cause, have been excluded. Few children with a normal cerebrospinal fluid pathway have been tested; those who have been were investigated for suspected hydrocephalus.
TABLE 1

COMPOSITION OF SERIES OF 165 SATISFACTORY TESTS ON 101 VERIFIED CASES

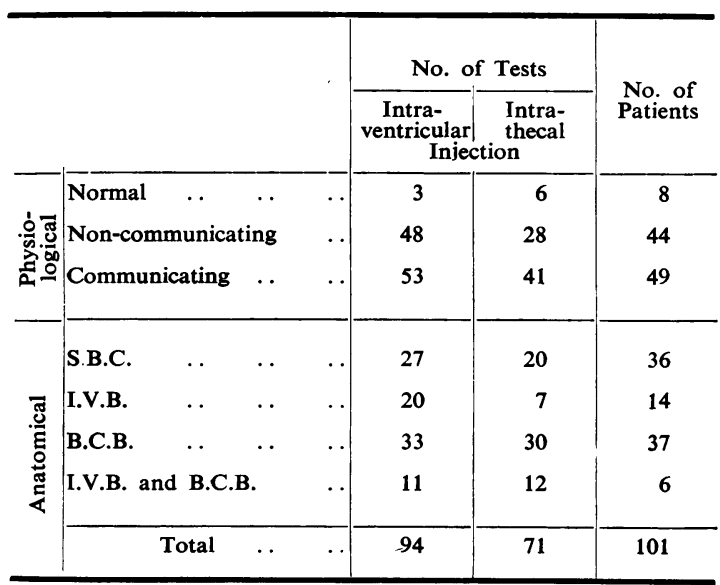

S.B.C. = spina bifida cystica with hydrocephalus.

I.V.B. = intraventricular block.

B.C.B. = basal cisternal block.

\section{Method}

A modification of Dandy and Blackfan's method has been used.

Injection of the Dye. The phenolsulphonphthalein* is supplied in ampoules containing $6 \mathrm{mg}$. of dye dissolved in $1 \mathrm{ml}$. of alkali. The whole content of an ampoule is neutralized immediately before use with an equal volume of $0.03 \%$ hydrochloric acid supplied. One millilitre ( $3 \mathrm{mg}$.) of the neutralized phenol red is then injected into a lateral ventricle or the lumbar theca and the dye is mixed with the cerebrospinal fluid by withdrawing the plunger of the syringe several times. The intraventricular injection is usually carried out three or four days after the intrathecal.

Collection of Urine. All the urine passed during the next 24 hours is then collected in divided specimens

* Messrs. Savory and Moore, Ltd., London, W.1, in boxes of six ampoules of dye and six of acid. Each batch is accurately matched. 
at, one, two, four, six, nine, 12,18 and 24 hours after injections of the dye. As the majority of the subjects are infants, it is advisable to catheterize females and to use Paul's tubing for the males. Care must be taken that no urine is lost, as this may considerably alter the result obtained.

Estimation. It was noticed that the pink dye in the urine faded slightly after 24 hours, especially if kept at room temperature. This is due to some constituent of the urine, for phenol red in water remains unaltered for days or weeks. The urine sample should therefore be stored in a refrigerator, and the dye content estimated within 24 hours of being passed.

The whole of each specimen is rendered alkaline with $10 \%$ sodium hydroxide until the intensity of the colour ceases to increase. The volume is then noted. About $10 \mathrm{ml}$. of the alkaline urine is centrifuged, and the colour intensity of the supernatant fluid is estimated in the photoelectric colorimeter with a No. 604 Ilford filter, using centrifuged alkaline urine as a blank and phenol red as a standard.

The phenol red standard is prepared as follows:

The contents of one ampoule (6 mg. of dye in $1 \mathrm{ml}$.) are made up to $10 \mathrm{ml}$. with distilled water to prepare a stock solution, which will keep two weeks or more in a refrigerator. Just before, $0.5 \mathrm{ml}$. of the stock phenol red solution is then made up to $100 \mathrm{ml}$. with centrifuged alkaline urine. This will keep for six hours.

Calculation of the Result. The dye content of each specimen (in $\%$ of the total injected)

$$
=\frac{\text { reading of test } \times \text { volume }}{\text { reading of standard } \times 10}
$$

A histogram such as the one shown in Fig. 1 can then be prepared and the total amount of dye excreted in the first six hours calculated.

In order to obtain reliable results, the full cooperation of the ward staff is absolutely essential. All urine losses must be reported accurately; the child must have a good urinary output and anaesthetics must be avoided.

The absorption of dye and the subsequent excretion in the urine should be a smooth process, however slow.

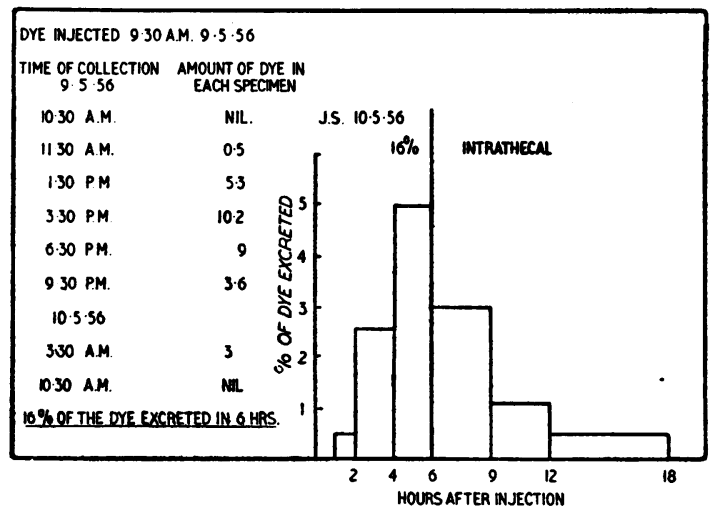

FIG. 1.-Specimen dye excretion results and histogram.
Erratic excretion results indicate that something has gone wrong with the test. This may be due to faulty urine collection or to erratic or suppressed urine production resulting from dehydration, vomiting or recent anaesthesia. To detect some of these errors, urine collection is continued for the whole 24-hour period, rather than the minimum six hours.

Repeated tests on the same patients under similar conditions show that the results are reproducible. When the test is carried out after air studies, the presence of air in the cerebrospinal fluid pathway does not seem to alter the amount of dye excreted. The children tolerate this form of investigation well. A few reactions have occurred, usually in the form of a transient pyrexia. There were, however, two cases of chemical meningitis and one of cortical vein and sinus thrombosis. The severity of these reactions compare favourably with those that follow air studies on hydrocephalic patients, who are known to be easily upset by any form of interference.

\section{Results}

To obtain full information with this test both intraventricular and intrathecal dye injection should be carried out. The results are best considered under their various anatomical groups (Fig. 2).

(1) Normal Cerebrospinal Fluid Pathway. Only eight children of the series belong to this group. The values obtained after intraventricular are considerably less than those after intrathecal injection. Dye injected into the lateral ventricle, even in a normal ventricular system, is considerably diluted and it then has to flow out into the subarachnoid space before it is absorbed. When the injection is made into the theca, little dilution takes place and absorption begins at once. This accounts for the dye excretion of above $25 \%$ after intraventricular, and above $40 \%$ after intrathecal, injection in the normal group.

(2) Basal Cistern Block. In these cases, the cerebrospinal fluid is able to reach the subarachnoid space, but is prevented by adhesions from going beyond the basal cisterns.

The results obtained after injection into both sites are below normal, but always more than $5 \%$. The ventricular dye result appears to bear little relation to the level of the basal cistern block, but is more an index of the degree of dilatation of the ventricles and the stasis of the cerebrospinal fluid. In the small number of cases in which really accurate ventriculograms are available the amount of dye excreted after intrathecal injection shows a close correlation with the level of the block within the basal cisterns.

(3) Intraventricular Block. As in this group the dye injected into the lateral ventricles is prevented 
ANATOMICAL SUBDIVISION

INTRAVENTRICULAR

INTRATHECAL
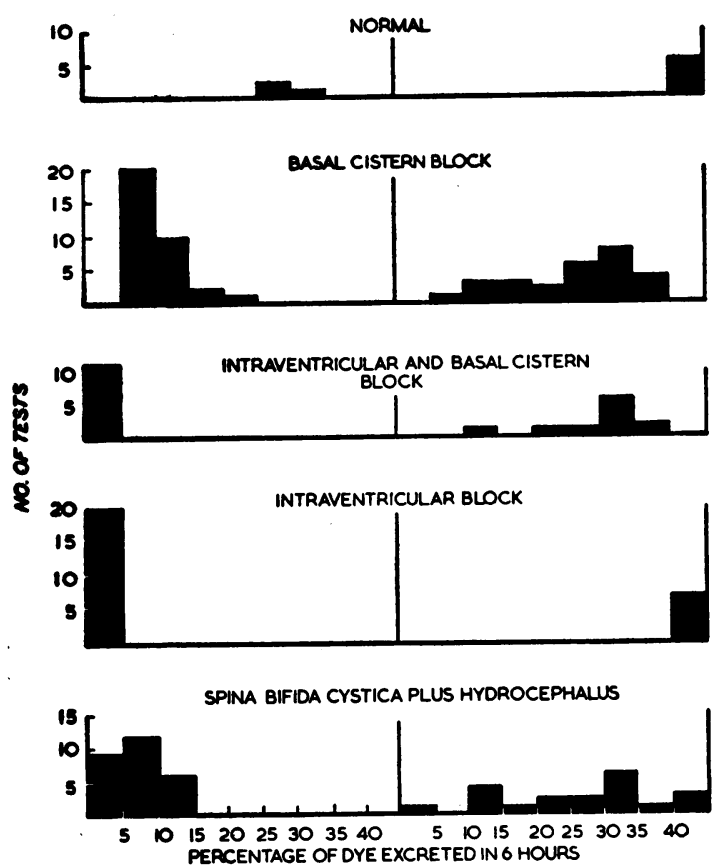

FIG. 2.-Tabulation of the results according to whether a communication did or did not exist. The amount of dye excreted in six hours is plotted horizontally, the number of tests in each $5 \%$ dye excretion group vertically. Intraventricular dye excretion results are on the left, intrathecal on the right.

from reaching the subarachnoid space, where it is normally absorbed, it is not possible to distinguish between blocks at any particular level within the ventricular system. Therefore all types of intraventricular block have to be grouped together. Cases with a normal subarachnoid space can, however, be separated from those which have basal cistern block in addition.

INTRAVENTRICUlaR BLOCK WITH NoRMAL SubARACHNOID SPACE. Here the block is generally at the level of the aqueduct of Sylvius, and is often a congenital malformation. The excretion figures after injection into the ventricle are always below $5 \%$ and often $1 \%$ or less, and the dye continues to be excreted in small amounts for a long time. The results after intrathecal injection are similar to those cases with a normal cerebrospinal fluid pathway.

INTRAVENTRICUlaR WITH Basal Cistern BLOCK. This combined lesion usually results from an infective process. The excretion results after intraventricular injection of dye are indistinguishable from cases which have only an intraventricular block. The values after intrathecal injections, however, are below normal as in cases with a basal cistern block.

(4) Spina Bifida Cystica and Hydrocephalus. This group is easily separated from the others on purely clinical grounds. In this condition the hydrocephalus may be due to a malformation at the level of the aqueduct or at the foramen magnum (ArnoldChiari malformation). Ascending infection from the spinal malformation may give rise to a spinal block, occlusion of the exit foramina of the fourth ventricle aqueduct block or a basal cistern block. As any combination of these lesions may be present in a particular case, a wide range of excretion results is obtained.

\section{Discussion}

The results are comparable with those of Dandy and Blackfan, and confirm some of their findings. Throughout their investigation these authors, however, obtained higher excretion values which produced less definite results.

In Fig. 3, when the findings of this investigation are tabulated according to the presence or absence of communication, there is considerable overlapping in the results of the two groups, as in Dandy and Blackfan's series, and no reliable conclusions can be

\section{PHYSIOLOGICAL SUBDIVISIONS}

INTRAVENTRICULAR INTRATHECAL

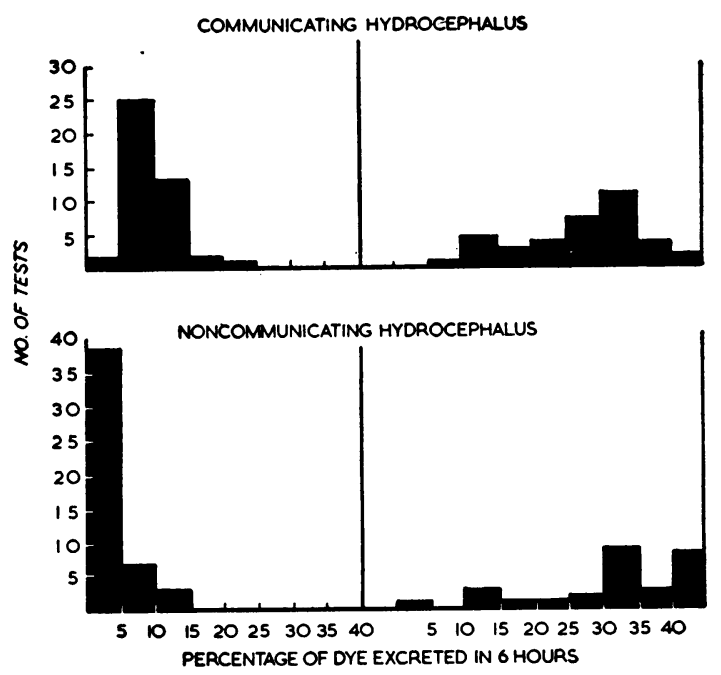

FIG. 3.-Tabulation of the results according to the anatomical site of the block. The amount of dye excreted in six hours is plotted horizontally, the number of tests in each $5 \%$ dye excretion group vertically. The intraventricular dye excretion results are on the left, intrathecal on the right. 
drawn. To account for this, Dandy and Blackfan suggested that cases with a 'partial' communication must exist.

If the results are tabulated according to the anatomical site of the block, however, clear-cut groups with distinct dye excretion patterns emerge. Conversely from the dye excretion patterns, the anatomical site of the block can be deduced. In the groups associated with spina bifida cystica, very variable excretion results are obtained for reasons which have already been given. Even here information, often difficult to obtain by other methods, can be deduced regarding the position of the obstructions and the state of the subarachnoid space. In the cases with combined ventricular and basal cisternal block, where the ventriculogram will demonstrate the site of the intraventricular block only, dye studies will give information about the basal cisterns as well. Thus there is no need for a difficult lumbar encephalogram. This is important when a ventriculocisternostomy is to be performed, an operation which could not succeed when the basal cisterns are impermeable.

Guthkelch (1955) claimed that dye tests in hydrocephalus were misleading. This may be true of the simple dye test for communication, but not of the phenol red dye excretion test when it is carefully performed. This test is not intended to replace good air encephalography but is likely to prove a really useful ancillary investigation, and of value when the $x$-ray pictures are not entirely reliable, or when adequate facilities are not available.

The test has been found useful in assessing the results of operation by comparing excretion values before and after. An adaptation of the method is being used with considerable success to select patients for a new form of ventriculo-subdural drainage (Forrest, Laurence and Macnab, 1957). It is hoped that eventually high excretion rates will be of value in predicting whether a case is likely to arrest naturally or not.

\section{Summary}

The method of the urine phenolsulphonphthalein excretion test as used in hydrocephalic children is described. Distinct dye excretion patterns were obtained in 101 patients, corresponding to the anatomical site of the blocks. The test was found of value as an ancillary to good pneumoencephalography; it could replace poor radiographs and gave additional information when there is a combined ventricular and basal cisternal block. The test may prove to be of prognostic value.

Dr. A. L. Woolf carried out the preliminary tests. I wish to thank the staff of The Hospital for Sick Children and of the Westminster Children's Hospital, and especially Mr. G. H. Macnab and Dr. M. Bodian, for their cooperation and advice; Miss $M$. Shorts and Miss B. Glashier for their technical assistance, Mr. G. Lyth for the charts, and the Research Committee of The Hospital for Sick Children for its financial support.

\section{REFERENCES}

Dandy, W. E. (1919). Ann. Surg., 70, 397.

- and Blackfan, K. D. (1914). Amer. J. Dis. Child., 8, 406.

, D. M., Laurence, K. M. and Macnab, H. G. (1957). Lancet, $1,1294$.

Guthkelch, A. N. (1955). Proc, roy. Soc. Med., 48, 849.

Heuer, G. J. and Dandy, W. E. (1916). Bull. Johns Hopk. Hosp., 